\title{
Machine learning prediction of thermodynamic and mechanical properties of multicomponent Fe-Cr-based alloys
}

\author{
B. O. Mukhamedov $\odot,{ }^{1, *}$ K. V. Karavaev ${ }^{2}$ and I. A. Abrikosov ${ }^{1}$ \\ ${ }^{1}$ Theoretical Physics Division, Department of Physics, Chemistry and Biology (IFM), Linköping University, SE-581 83, Linköping, Sweden \\ ${ }^{2}$ Materials Modeling and Development Laboratory, National University of Science and Technology “MISIS,” Moscow 119049, Russia
}

(Received 23 July 2021; accepted 21 September 2021; published 18 October 2021)

\begin{abstract}
We apply machine learning algorithms to optimize thermodynamic and elastic properties of multicomponent $\mathrm{Fe}-\mathrm{Cr}$ alloys with additions of $\mathrm{Ni}, \mathrm{Mo}, \mathrm{Al}, \mathrm{W}, \mathrm{V}$, and $\mathrm{Nb}$. The target properties are mixing enthalpy, Young's elastic modulus, and the ratio between shear and bulk moduli, which is often used as a phenomenological criterion for a material's ductility. We thoroughly analyze the descriptors that provide the robust performance of the machine learning models. Next, the iterative active learning method is used for the optimization of the chemical composition to simultaneously improve both thermodynamic stability and the elastic properties of Fe-Cr-based alloys. As a result, we predict compositions of thermodynamically stable alloys with improved mechanical properties, demonstrating the high potential of data-driven computational design in the field of materials for nuclear energy applications.
\end{abstract}

DOI: 10.1103/PhysRevMaterials.5.104407

\section{INTRODUCTION}

Fe-Cr-based bcc alloys are widely used as important structural and industrial steels. They are known for their resistance to corrosion and irradiation-induced swelling at high temperatures [1]. This makes the Fe-Cr-based steels a suitable candidate material for harsh environments in, for example, nuclear and fusion reactors. At the same time, increasing demands for materials performance, e.g., for the next generation of nuclear reactors, call for a development of novel steels. One path to that is the use of multicomponent alloying. At the same time, increasing the number of alloy components could greatly increase the complexity of purely experimental materials design, leading to longer development time and higher costs. Theoretical predictions, which take advantage of state-of-the-art first-principles simulations combined with alternative data-driven methods, such as machine learning, are believed to significantly accelerate discovery of novel materials and shorten the time it takes to bring them to practical applications [2].

Considering the phase stability of $\mathrm{Fe}$-Cr-based steels, which is perhaps the main criterion for a construction material, the situation is quite delicate. The binary $\mathrm{Fe}-\mathrm{Cr}$ phase diagram has a miscibility gap showing that alloys decompose spinodally into Fe- and Cr-rich phases at low and medium temperatures. At the same time, the number of density func-

\footnotetext{
*boburjon.mukhamedov@liu.se
}

Published by the American Physical Society under the terms of the Creative Commons Attribution 4.0 International license. Further distribution of this work must maintain attribution to the author(s) and the published article's title, journal citation, and DOI. Funded by Bibsam. tional theory (DFT) studies suggested that diluted alloys with up to $\sim 6-8$ at. $\% \mathrm{Cr}$ demonstrate an anomalous thermodynamic stability with negative mixing enthalpy [3-8]. This anomalous stability of $\mathrm{Fe}-\mathrm{Cr}$ alloys is related to the magnetic behavior of $\mathrm{Cr}$ atoms, which have the magnetic moments aligned antiparallel to the $\mathrm{Fe}$ moments due to antiferromagnetic exchange interactions between $\mathrm{Fe}-\mathrm{Cr}$ pairs in the first nearest neighborhood. The $\mathrm{Cr}-\mathrm{Cr}$ pairs in the first nearest neighborhood also have antiferromagnetic exchange interactions; thus, the high $\mathrm{Cr}$ concentrations lead to the frustration of local magnetic configuration and gradually reduce the magnetic moments on $\mathrm{Cr}$ atoms. As a result, the mixing enthalpy of $\mathrm{Fe}-\mathrm{Cr}$ alloys increase with $\mathrm{Cr}$ content. In addition to the mixing enthalpy, calculated bulk modulus and lattice parameters of $\mathrm{Fe}-\mathrm{Cr}$ alloys also show abnormal behavior at the low-Cr region: The lattice parameter changes with the maximum in the range of $0-8$ at. $\% \mathrm{Cr}$, while the bulk modulus has a minimum.

Despite the importance of Fe-Cr-based alloys and a large number of first-principles simulations carried out for this system, the use of data-driven methods for predicting properties of novel alloys has been quite limited. Ponomareva et al. [9] have used the exact muffin-tin orbitals (EMTO) method combined with the coherent potential approximation (CPA) to simulate the effect of multicomponent alloying with $\mathrm{Ni}$, $\mathrm{Mo}, \mathrm{Al}, \mathrm{W}, \mathrm{V}$, and $\mathrm{Nb}$ on the thermodynamic and mechanical properties of $\mathrm{Fe}-\mathrm{Cr}$-based bcc alloys and to contribute to a database for the design of materials for nuclear energy applications [10]. Besides the generated data, the study has shown a correlation between the mixing enthalpy and the value of the local moment on $\mathrm{Fe}$ atoms in the alloys: The elements that reduce the Fe magnetic moment increase the stability of alloys. Alloying with $\mathrm{Al}$ has been predicted to provide the highest stabilizing effect on the binary $\mathrm{Fe}-\mathrm{Cr}$ alloys, while nickel has destabilized the solid solutions. An addition of 
1 wt $\%$ of $\mathrm{Nb}, \mathrm{W}$, or Mo has decreased the stability of the alloys. The calculations of elastic properties have shown that $\mathrm{Cr}$ should have the highest contribution to the elastic moduli. On the other hand, when the $\mathrm{Cr}$ content becomes higher than $\sim 9$ wt $\%$ the mixing enthalpy has turned positive. Moreover, the addition of $\mathrm{Al}$ to the $\mathrm{Fe}-\mathrm{Cr}$ alloys has increased the elastic moduli, while Ni has an opposite effect [9].

In the present work we advance our study on multicomponent $\mathrm{Fe}-\mathrm{Cr}$ alloys by applying the machine learning (ML) algorithms for high-throughput prediction of the thermodynamic and elastic properties of $\mathrm{Fe}-\mathrm{Cr}$ alloys. We used the dataset of 186 alloys from Ref. [9] to perform original training of machine learning models that can predict the mixing enthalpy $H_{\mathrm{mix}}$, Young's modulus $E$, and the ratio between shear and bulk moduli $G / B$. The latter is considered as a phenomenological criterion for material's ductility. Next, we used an iterative active learning approach for further optimization of the chemical composition of Fe-Cr-based alloys in order to simultaneously improve their thermodynamic stability and elastic properties.

\section{METHODOLOGY}

\section{A. Machine learning algorithms}

The quantitative prediction of the target property $\hat{y}(x)$ is the regression problem, which can be solved using the supervised ML algorithms. In this work, we considered two ML algorithms: artificial neural network and support vector machines (or support vector regression).

The artificial neural network (ANN) is built as a series of interconnected artificial neurons to mimic the work of the human brain [11]. The ANN consists of three types of layers: (1) the input layer; (2) hidden (or computational) layers; (3) output layer. The input layer passes the data of the descriptor directly to the first hidden layer. In the hidden layers, each neuron calculates the weighted sum of the input signal coming from the previous layer and adds the bias. Then the activation function defines the output signal passed to the next layer of neurons. The output layer produces the final result, i.e., the prediction of the target property. In this work, the ANN contains three hidden layers with 20, 50, and 20 neurons, respectively. The rectified linear unit function (ReLU) was used as an activation function for each neuron. During the training of ANN the weights and biases of neurons are adjusted to minimize the prediction error. We used TENSORFLOW software [12] to build the ANN models.

Support vector machines (or support vector regression, SVR) are the supervised learning algorithms, which use the kernel function to find a hyperplane and transform the descriptors into higher-dimensional space [13]. With a kernel function, the continuous output $\hat{y}(x)=f(x)$ from a set of features $x$ is given by

$$
f(x)=\langle w, K(x)\rangle+b,
$$

where $K(x)$ is the kernel function; $w$ and $b$ are determined via minimization of the penalty function:

$$
\frac{1}{2}\|w\|^{2}+C \sum_{i=1} L\left[f\left(x_{i}, y_{i}\right)\right],
$$

where $C$ is the penalty parameter and $L$ is the loss function:

$$
L\left[f\left(x_{i}, y_{i}\right)\right]=\max \left\{\left|f\left(x_{i}\right)-y_{i}\right|\right\} .
$$

As a kernel function in this work, we considered the radial basis function $(\mathrm{RBF})$ which is given by

$$
K\left(x_{i}, x_{j}\right)=\exp \left(-\gamma\left\|x_{i}-x_{j}\right\|^{2}\right) .
$$

The fitting parameters $C$ and $\gamma$ are optimized to improve the performance of SVR models. In this work, the SVR models were trained within the SCIKIT-LEARN code [14].

\section{B. Data collection and preprocessing}

As the training dataset for ML models, we use the thermodynamic data for multicomponent $\mathrm{Fe}-\mathrm{Cr}$ alloys from Ref. [9] The original set contains data for 186 alloys with the composition of elements in the following range (wt \%): $0 \leqslant \mathrm{Cr} \leqslant$ $20,0 \leqslant \mathrm{Ni} \leqslant 20,0 \leqslant \mathrm{Mo} \leqslant 1,0 \leqslant \mathrm{~V} \leqslant 1,0 \leqslant \mathrm{~W} \leqslant 1,0 \leqslant$ $\mathrm{Nb} \leqslant 2,0 \leqslant \mathrm{Al} \leqslant 5$, and $\mathrm{Fe}$ is a principal element.

One of the important tasks in a machine learning approach is the construction of descriptor $x$, a subset of features that properly predict the target property $y$. In this work the target properties are the Young's modulus $E$, the mixing energy $H_{\mathrm{mix}}$, and Pugh's ratio $G / B$. Since the goal of this work is to improve the target properties via optimization of chemical composition one needs to choose the features that do not require any additional DFT calculations. Thus, as the candidates we considered five empirical features: the atomic radius mismatch $\delta$, average atomic volume $V$, valence electron concentration $C_{\mathrm{vec}}$, total electronegativity $\chi$ in Pauling's scale, and mixing entropy $S_{\text {mix }}$ :

$$
\begin{gathered}
\delta=\sqrt{\sum_{i} c_{i}\left(1-\frac{r_{i}}{\bar{r}}\right)^{2},} \\
V=\sum_{i} c_{i} \frac{4}{3} \pi r_{i}^{3}, \\
\chi=\sum_{i} c_{i} \chi_{i}, \\
C_{\mathrm{vec}}=\sum_{i} c_{i} C_{\mathrm{vec}, i}, \\
S_{\text {mix }}=-R \sum_{i} c_{i} \ln \left(c_{i}\right),
\end{gathered}
$$

where $c_{i}$ denotes the concentration of the $i$ th element in the alloy; $r_{i}$ is the atomic radius and $\bar{r}$ is the corresponding average atomic radius; $\chi_{i}$ and $C_{\mathrm{vec}, i}$ denote electronegativity and valence electron concentrations for the $i$ th component; $R$ is the universal gas constant.

We should note that $S_{\text {mix }}$ depends only on chemical composition and the number of elements in the alloy, while the four other features $\delta, V, \chi$, and $C_{\mathrm{vec}}$ are calculated as a concentration average of corresponding elemental and empirical values. In Sec. III A we will demonstrate that the five-feature descriptor $\left\{\delta, V, \chi, C_{\mathrm{vec}}, S_{\mathrm{mix}}\right\}$ is certainly sufficient to train the robust models for $H_{\text {mix }}$, elastic modulus $E$, and the $G / B$ ratio. The features selected in this work were previously used in several studies to build the models for predictions of the phase separation and elastic properties of solids [15-20]. For example, Huang et al. [19] used the features $\delta, C_{\mathrm{vec}}, H_{\mathrm{mix}}$, 

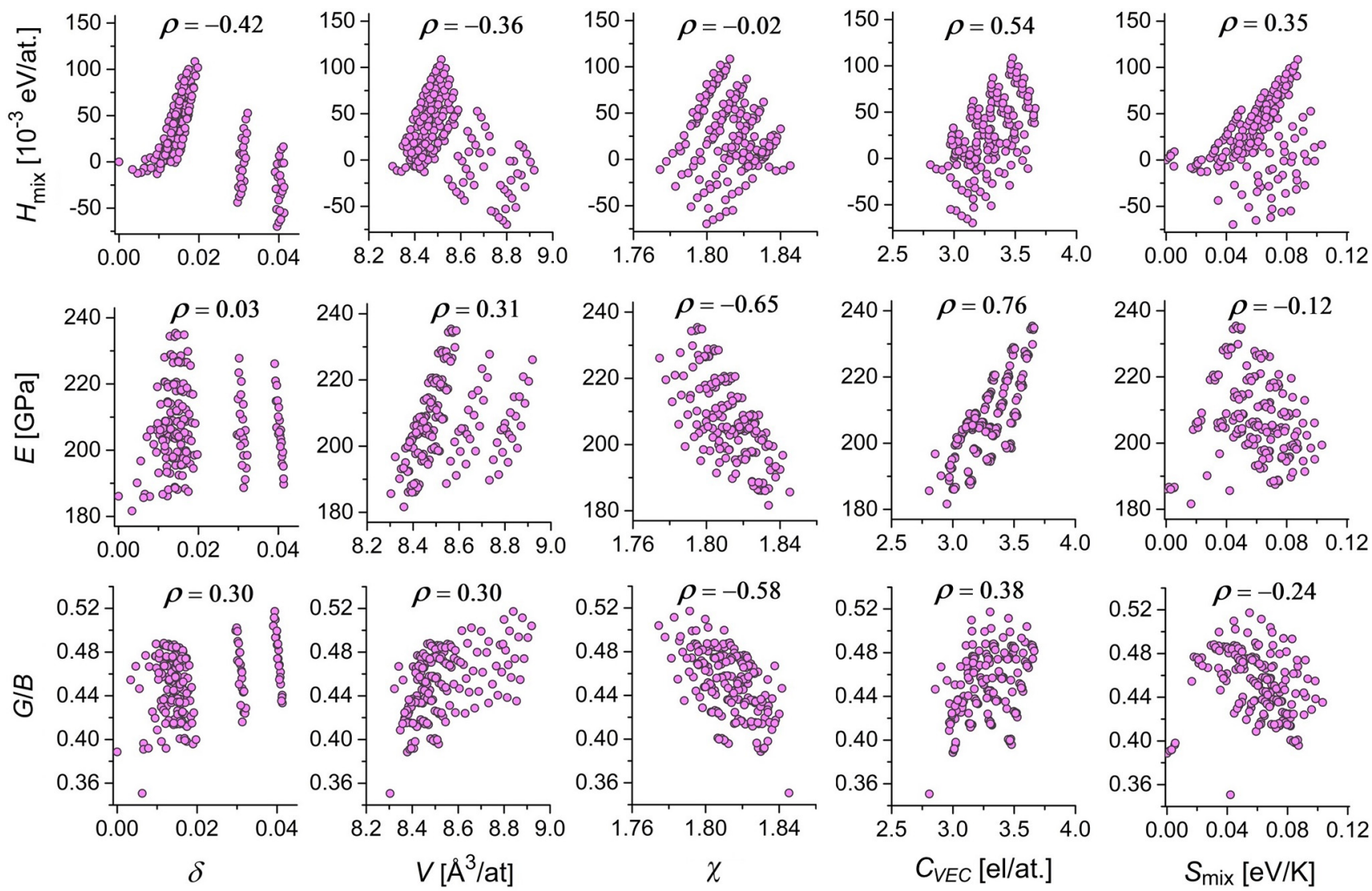

FIG. 1. Three target properties $E, H_{\text {mix }}, G / B$ plotted against five features $\delta, V, C_{\text {vec }}, \chi$, and $S_{\text {mix }}$ selected for descriptor in this study. Corresponding correlation coefficients are given on top of each scatter plot. See text for more discussion.

$S_{\text {mix }}$, and relative electronegativity $\Delta \chi$ to successfully predict the phase separation between high-entropy alloys and intermetallics. The authors also claim that features $\delta$ and $C_{\text {vec }}$ are more crucial for their task in comparison to $H_{\text {mix }}, S_{\text {mix }}$, and $\Delta \chi$ [19]. In Ref. [20], the combination of features $\delta, V, C_{\mathrm{vec}}$, $\chi$, and $S_{\text {mix }}$ was used to fit ML models that predict the bulk and shear moduli of metallic glasses. It is widely accepted that electronegativity difference $\Delta \chi$ is an important parameter for alloy formation and charge transfer effects [21-23]. However, in the present work we did not see any strong correlation between $\Delta \chi$ and target properties. Therefore, $\Delta \chi$ was not included in the descriptor.

In Fig. 1 we show the pair plots and the Pearson's correlation coefficients $\rho$ between five features and three target properties. The Pearson's coefficients $\rho$ represent the strength of linear correlation between two parameters. The $C_{\text {vec }}$ demonstrates the highest correlation with $H_{\text {mix }}$ and $E$, while $\chi$ has $|\rho|>0.5$ with $E$ and $G / B$. In case of the $G / B$ ratio, the strongest correlation, $\rho=-0.58$, belongs to the average electronegativity $\chi$; and the other features demonstrate weaker correlations $|\rho|<0.5$. The weakest correlations $\rho$ are observed between $\delta$ and $E$, and between $S_{\text {mix }}$ and $E$. However, we should note that the plots of target properties $E, H_{\text {mix }}$, and $G / B$ against $\delta$ and $V$ are nontrivial: for each plot with $\delta$ and $V$ there are three clearly distinguishable groups of scatters, which separately might have stronger correlation with corresponding target properties. Thus, in the case of features $\delta$ and $V$ the Pearson's coefficient $\rho$ may not fully represent their importance. Overall, Fig. 1 shows that there is no strong enough pair correlation between any single feature and target properties. This means that it is impossible to accurately predict $E, H_{\text {mix }}$, and $G / B$ by using only one particular feature. Thus, we suggest considering all possible combinations of features and varying the number of features in the descriptor from 1 to 5 .

The normalization of the descriptor is required when the features have a different range of values. After normalization, the range of values of the features becomes of the same order. The normalization of the descriptors was carried out using the following formula:

$$
x^{\prime}=\frac{x-\bar{x}}{\sigma_{x}},
$$

where $\bar{x}$ is the mean value of the feature; $\sigma_{x}$ is the standard deviation from the mean value $\bar{x}$.

It should be noted that the normalization of features must be carried out both for the training and the test sets of data.

\section{Cross validation}

To estimate the performance of ML models employed in the present study we used the $k$-fold cross-validation $(\mathrm{CV})$ method [24]. In this method the dataset is split into $k$ equal sized folds (where $k$ is an integer) and each $k$ th fold is used as a validation set, while the remaining $k-1$ parts of the dataset are used to fit the model. The mean absolute error $\Delta_{M A E}$ 


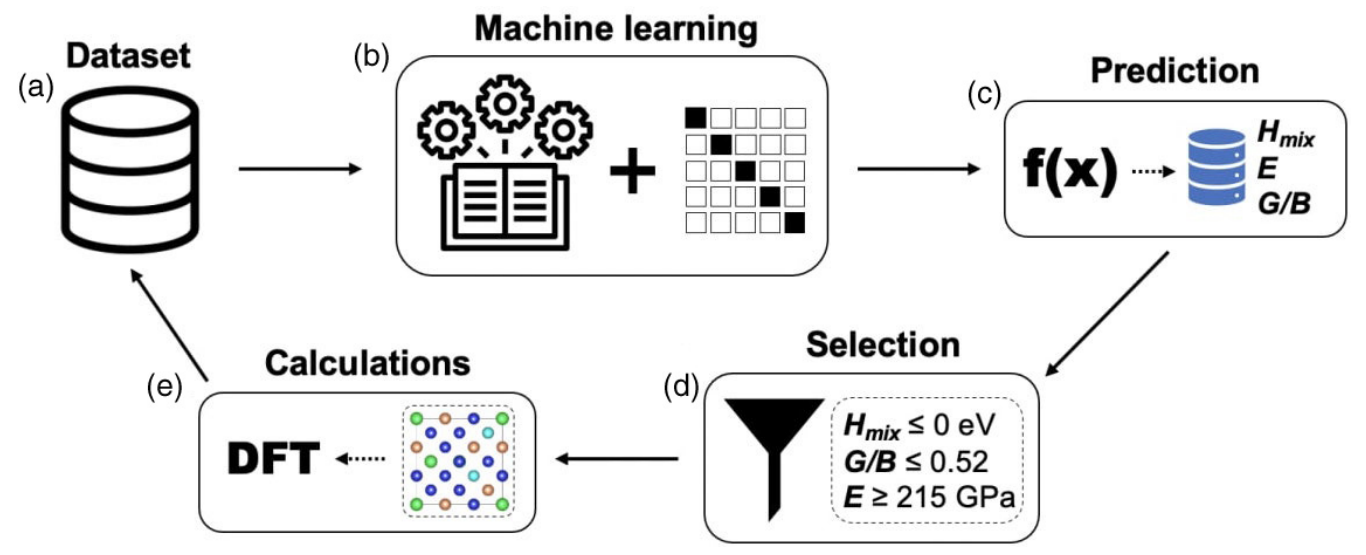

FIG. 2. A schematic representation of the iterative active learning process adopted in the present study. The iterative loop involves (a) data collection; (b) fitting the ML models and cross validation; (c) predicting the target properties $H_{\text {mix }}, E$ modulus, $G / B$ ratio for virtual space of alloys; (d) selection of promising candidates; (e) DFT calculations for four randomly selected compositions among candidates. The iterations continue until there is no further improvement in target properties $E, H_{\text {mix }}$, and $G / B$.

and determination parameter $R^{2}$ were used to characterize the prediction error and accuracy:

$$
\begin{gathered}
\Delta_{M A E}=\frac{1}{N} \sum_{i=1}^{N}\left(y_{i}-\hat{y}_{i}\right), \\
R^{2}=1-\frac{\frac{1}{N} \sum_{i=1}^{N}\left(y_{i}-\hat{y}_{i}\right)^{2}}{\frac{1}{N} \sum_{i=1}^{N}\left(y_{i}-\bar{y}\right)^{2}},
\end{gathered}
$$

where $N$ is the sample size, $\hat{y}_{i}$ is the predicted value, and $y_{i}$ is the actual value.

Within this method the validation process is repeated $k$ times. In this work we considered $k=5$. The average value of recorded errors defines the cross-validation error $\left(\Delta_{C V E}\right)$. In a similar way one defines the cross-validation accuracy $\left(R_{C V A}^{2}\right)$ of the predictions. As a first step in the $\mathrm{CV}$ it is required to randomly shuffle the dataset.

\section{Active learning}

Once the ML models for the $E$ modulus, $H_{\text {mix }}$, and $G / B$ ratio of the alloys comprising the original dataset have been trained one can search for compositions to simultaneously improve the Young's modulus and the hermodynamic stability of multicomponent alloys. For this purpose, we generate the virtual space of alloys with the following range of concentrations in steps of $1 \mathrm{wt}$ $\%: \quad 50 \leqslant \mathrm{Fe} \leqslant 100,0 \leqslant \mathrm{Cr} \leqslant 25,0 \leqslant \mathrm{Ni} \leqslant 25,0 \leqslant$ Mo $\leqslant 2,0 \leqslant \mathrm{~V} \leqslant 2,0 \leqslant \mathrm{~W} \leqslant 2,0 \leqslant \mathrm{Nb} \leqslant 2$, and $0 \leqslant \mathrm{Al} \leqslant 10$. The virtual space contains 556578 alloys in total, and its composition range is slightly wider compared to the original dataset. Next, we calculate five features $\delta$, $V, \chi, C_{\mathrm{vec}}$, and $S_{\text {mix }}$ for all the alloys in virtual space and predict their target properties $E, H_{\text {mix }}$, and $G / B$. Note that the normalization of the feature subset $x$ for virtual space must be done by using the same $\bar{x}$ and $\sigma_{x}$ as for the training set of original data [see Eq. (10)].

Next, we select promising candidates with $H_{\text {mix }} \leqslant 0 \mathrm{eV} /$ at, $G / B \leqslant 0.52$, and $E \geqslant 215 \mathrm{GPa}$, i.e., the candidates that are thermodynamically stable with respect to a decomposition into pure alloy components, nonbrittle according to the phenomenological Pugh's ratio and have higher elastic modulus $E$ compared to the calculated value of $E$ among the stable alloys $\left(H_{\text {mix }} \leqslant 0\right)$ in the original dataset. Once the selection criteria were applied to the virtual alloys, we randomly choose four compositions among the promising candidates and perform for them first-principles calculations of $E, H_{\mathrm{mix}}$, and $G / B$. Then, we add these compositions to the original dataset and retrain the ML models. In this way one improves the performance of the models with new data if the previous predictions were not sufficiently accurate. The sequence described above defines one loop of the active learning process. The iterations stop once there is no further improvement of target properties, i.e., that is there is no more increase in elastic modulus $E$ among the alloys with $H_{\text {mix }} \leqslant 0 \mathrm{eV} /$ at. and $G / B \leqslant 0.52$. The full process is schematically shown in Fig. 2.

\section{E. DFT calculations}

Electronic-structure calculations for $\mathrm{Fe}$-Cr-based alloys that are not present in the original dataset of Ref. [9] were carried out at temperature $T=0 \mathrm{~K}$ in the framework of the exact muffin-tin orbitals method combined with the coherent potential approximation (EMTO-CPA) to model the substitutional chemical disorder [25,26]. The computational setup is exactly as in Ref. [9] to ensure the compatibility of the data. To summarize the most important details, the self-consistent charge densities were obtained within the local-spin-density approximation (LSDA) [27], while the total energies were calculated within the generalized gradient approximation (GGA) [28]. Integration in the reciprocal space was performed over a grid of $37 \times 37 \times 37 k$ points. The energy integration was carried out in the complex plane using a semielliptic contour comprising 24 energy points. Calculations were performed for a basis set including valence $s p d f$ orbitals. We have considered collinear alignment of magnetic moments. The elastic constants were defined from the energy-strain relationship according to Ref. [25]. The polycrystalline elastic moduli were obtained using Voigt-Reuss-Hill averaging [29-31]. 

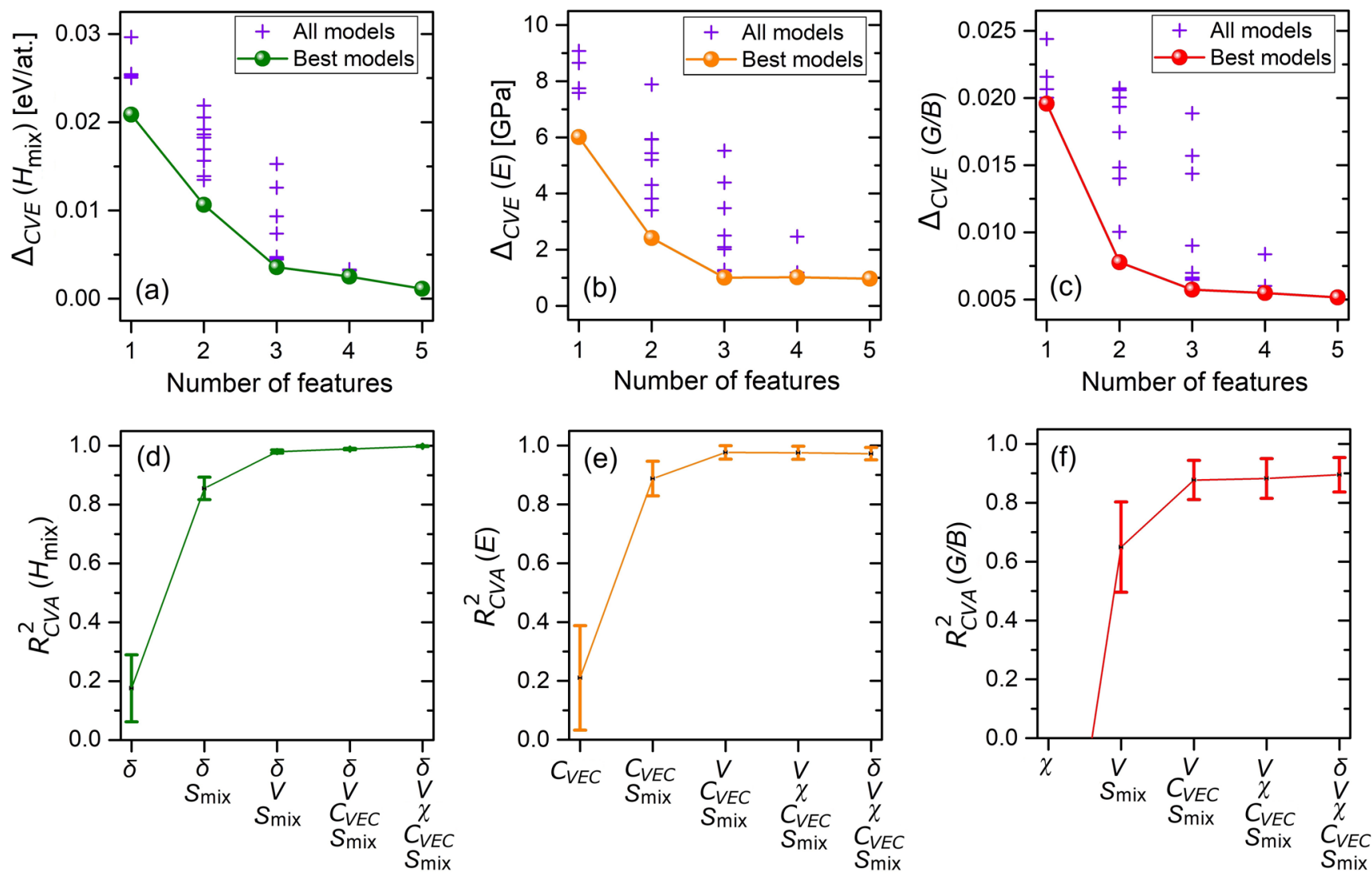

FIG. 3. Fivefold $\Delta_{C V E}$ calculated for all three target properties (a)-(c) when the different subsets of features were included in the fitting process: The crosses represent all possible models when the number of features in the descriptor varies from 1 to 5 . The solid lines correspond to the best models (the lowest $\Delta_{C V E}$ ) for a given number of features. The corresponding fivefold cross-validation accuracy $R_{C V A}^{2}$ of the best models (d)-(f). The horizontal axis demonstrates the feature subsets corresponding to the best models. Solid lines represent the mean accuracy of fivefold CV for a given number of features.

\section{RESULTS}

\section{A. Machine learning}

In Fig. 3 we show the performance of SVR models fitted against each possible subset of features, i.e., when the number of features in the descriptor varies from 1 to 5 . The $k$-fold CV test was carried out for each possible model. In Figs. 3(a)3(c), all three target properties $H_{\text {mix }}, E$, and $G / B$ demonstrate the lowest $\Delta_{C V E}$ once all five features are included in the fitting of the SVR models. The solid lines in Figs. 3(a)-3(c) correspond to the best models for a given number of features in the descriptor. One can see that the best models with threeand four-feature subsets also demonstrate low values of the $\Delta_{C V E}$. However, we should note that the $\Delta_{C V E}$ does not fully reflect the prediction capabilities of the models. Thus, we also calculated the prediction accuracy for the best models.

Figures 3(d)-3(f) show the cross-validation accuracy $R_{C V A}^{2}$ of the best models for a given number of features in the descriptor. The subsets of features $\left\{\delta, V, S_{\text {mix }}\right\},\left\{\delta, V, C_{\text {vec }}\right.$, $\left.S_{\text {mix }}\right\}$, and $\left\{\delta, V, \chi, C_{\text {vec }}, S_{\text {mix }}\right\}$ allow one to properly predict $\left(R_{C V A}^{2}>0.9\right)$ the mixing energy $H_{\text {mix }}$ of Fe-Cr-based alloys. The total Pauling's electronegativity $\chi$ has the least effect on the prediction of $H_{\mathrm{mix}}$. It is not surprising since we already demonstrated in Fig. 1 that $H_{\text {mix }}$ has the weakest correlation with $\chi$. However, once $\chi$ is included in the SVR model the prediction error $\Delta_{C V E}$ for $H_{\text {mix }}$ decreases [see Fig. 3(a)] from
0.004 to $0.0015 \mathrm{eV} / \mathrm{at}$, and the latter one is very close to $10^{-3} \mathrm{eV} / \mathrm{at}$, which is considered as high accuracy for the first-principles calculations of the total energy.

As for the Young's modulus, the high performance of the SVR models $\left(R_{C V A}^{2}=0.972\right.$ and $\left.\Delta_{C V E}=1.78 \mathrm{GPa}\right)$ can be achieved even with a three-feature descriptor $\left\{V, C_{\mathrm{vec}}, S_{\mathrm{mix}}\right\}$. Moreover, by adding the atomic size mismatch $\delta$ and total electronegativity $\chi$ into the descriptor one can slightly improve the predictions: For the model trained with descriptor $\left\{\delta, V, \chi, C_{\mathrm{vec}}, S_{\mathrm{mix}}\right\}$ the $R_{C V A}^{2}=0.977$ and $\Delta_{C V E}=1.55 \mathrm{GPa}$. Despite the good correlation between $\chi$ and elastic modulus $E$, the $\chi$ does not work well in combination with other features in the subsets. For example, one would expect a good predictability of $E$ modulus from the model fitted against subset $\left\{V, \chi, C_{\mathrm{vec}}\right\}$; however, this model has a cross-validation error $\Delta_{C V E}=6.9 \mathrm{GPa}$ and demonstrates one of the worst performances among the three-feature models. On the other hand, by adding the mixing entropy $S_{\text {mix }}$ into the descriptor one can significantly improve the prediction of elastic modulus $E$. For example, by fitting $E$ against two features $\left\{C_{\mathrm{vec}}, S_{\mathrm{mix}}\right\}$ one can reach the $R_{C V A}^{2}=0.85$.

In the case of Pugh's ratio $G / B$, the best SVR model demonstrates the average accuracy $R_{C V A}^{2}=0.9$, which can be considered as satisfactory even though it is lower compared to the models for mixing energy $H_{\mathrm{mix}}$ and $E$ modulus. Similar to the case of $E$ modulus, the descriptor $\left\{V, C_{\mathrm{vec}}, S_{\text {mix }}\right\}$ provides 
TABLE I. Prediction error $\Delta_{C V E}$ and accuracy $R_{C V A}^{2}$ of ANN and SVR models for the Young's modulus $E$, mixing enthalpy $H_{\text {mix }}$, and $G / B$ ratio.

\begin{tabular}{|c|c|c|c|c|c|c|}
\hline \multirow[b]{2}{*}{ ML method } & \multicolumn{3}{|c|}{$R_{C V A}^{2}(\%)$} & \multicolumn{3}{|c|}{$\Delta_{C V E}$} \\
\hline & $H_{\text {mix }}$ & $E$ & $G / B$ & $H_{\text {mix }}\left(\mathrm{eV} /\right.$ at $\left.\times 10^{-3}\right)$ & $E(\mathrm{Gpa})$ & $G / B$ \\
\hline ANN & $99.5 \pm 0.1$ & $96.5 \pm 1.7$ & $84.8 \pm 10.3$ & $2.5 \pm 0.4$ & $2.1 \pm 0.4$ & $0.010 \pm 0.004$ \\
\hline SVR & $99.8 \pm 0.1$ & $97.3 \pm 2.1$ & $89.5 \pm 5.9$ & $1.5 \pm 0.2$ & $1.8 \pm 0.7$ & $0.009 \pm 0.003$ \\
\hline
\end{tabular}

low prediction error for the $G / B$ ratio, and $\Delta_{C V E}=0.01$. By adding the $\delta$ and $\chi$ into the descriptor one can slightly improve the SVR model. The best single-feature model demonstrates negative $R_{C V A}^{2}$, which shows that the fitting subset for the $G / B$ ratio was chosen poorly. According to Figs. 3(e) and 3(f) the mixing entropy $S_{\mathrm{mix}}$ is an important feature for all three target properties of multicomponent Fe-Cr-based alloys, so the models fitted on the subsets $\left\{\delta, S_{\mathrm{mix}}\right\},\left\{C_{\mathrm{vec}}, S_{\mathrm{mix}}\right\}$, and $\left\{V, S_{\mathrm{mix}}\right\}$ allow one to predict $H_{\mathrm{mix}}, E$, and $G / B$, respectively, with average accuracy $R_{C V A}^{2}=0.6-0.8$. However, once the models are fitted against the five-feature subset $\left\{\delta, V, \chi, C_{\mathrm{vec}}\right.$, $\left.S_{\text {mix }}\right\}$ one can observe the lowest $\Delta_{C V E}$ and the highest $R_{C V A}^{2}$ in the predictions of the $H_{\text {mix }}, E$, and $G / B$ ratio.

To analyze the effect of different combinations of features on the performance of ML models we used only the SVR method. A similar analysis as for the ANN method would be too cumbersome, since it requires one to optimize the number of sublayers in the hidden layers and the number of neurons in the sublayers for every possible model. Therefore, to train the ANN models we used the five-feature subset $\left\{\delta, V, \chi, C_{\mathrm{vec}}\right.$, $\left.S_{\text {mix }}\right\}$, which provided the minimum $\Delta_{C V E}$ in the case of the SVR models. The performances of the SVR and ANN models are compared in Table I. In the case of $H_{\text {mix }}$ and $E$ modulus the difference in predictive ability between the SVR and ANN models is very small. Overall, SVR models with RBF kernel function demonstrate lower prediction error $\Delta_{C V E}$ and higher accuracy $R_{C V A}^{2}$ for all three target properties $H_{\text {mix }}, E$ modulus, and $G / B$ ratio. Thus, for further active learning studies we use the SVR-RBF models.

\section{B. Active learning}

Using the pretrained SVR models we can predict the Young's modulus $E$, mixing energy, and $G / B$ ratio for all the generated 556578 virtual $\mathrm{Fe}-\mathrm{Cr}$ alloys (see Supplemental Material [32]). Next, by applying the selection criteria $\left(H_{\text {mix }} \leqslant 0 \mathrm{eV} / \mathrm{at} ; G / B \leqslant 0.52\right.$ and $\left.E \geqslant 215 \mathrm{GPa}\right)$ we limit the search area for promising candidates. Given the fact that the trained SVR models for $H_{\text {mix }}, E$ modulus, and $G / B$ demonstrate high prediction accuracy, we randomly select four compositions among the candidates and perform for them new EMTO-CPA calculations. The iterative machine learning procedure was performed four times, and the models were updated with the new data after each iteration. In Table II, we list compositions of candidates and corresponding values of $H_{\text {mix }}, E$, and $G / B$.

In the first iteration, the randomly chosen candidates contain 4 wt $\%$ Al, 19-20 wt \% Cr, and 1-2 wt \% Nb. The calculated values of the $E$ for the candidates are larger than $215 \mathrm{GPa}$ and $G / B$ ratios are lower than 0.52 . In the case of $H_{\text {mix }}$, the actual values for three compositions out of four are positive while ML models predict negative $H_{\text {mix }}$. Thus, at the first iteration the model for $H_{\mathrm{mix}}$ is not sufficiently accurate. With the following iteration, the performance of the model for $H_{\text {mix }}$ becomes better. Figure 4 shows the ML predictions of target properties plotted against the actual values. Here, one can see that predictions of $E$ and $G / B$ are in good agreement with actual values after each iteration of the active learning. We note that the concentration of $\mathrm{Al}$ in candidates increases up to $9 \mathrm{wt} \%$ with iterations, and $\mathrm{Cr}$ increases up to $25 \mathrm{wt}$ $\%$. However, there is no significant improvement in elastic modulus $E$ and the highest value $\approx 227 \mathrm{GPa}$. According to the plots in Figs. 4(a) and 4(c), the SVR models for $E$ modulus and $G / B$ ratio demonstrate an excellent predictive ability for the compositions beyond the original dataset, even during the first iteration. The model for $H_{\text {mix }}$ becomes more reliable after the second iteration of the active learning.

Let us recall that the goal of this work was to predict the chemical composition of the multicomponent Fe-Cr-based alloys to simultaneously improve their elastic modulus and stability. As a result of the active learning, we can now identify an alloy with the composition $\mathrm{Fe}-25 \% \mathrm{Cr}-9 \% \mathrm{Al}$ as the most promising system. It demonstrates the lowest $H_{\text {mix }}=$ $-0.0247 \mathrm{eV} /$ at and $E=226.0 \mathrm{GPa}$. Of course, we note that $H_{\text {mix }}$ in this work is calculated with reference to the pure elements, which does not include the possible presence of competing intermetallic phases. This could become important

TABLE II. DFT calculations for the selected Fe-Cr-based candidates after each iteration of active learning.

\begin{tabular}{|c|c|c|c|c|c|c|c|c|c|c|c|}
\hline \multirow[b]{2}{*}{ Iteration } & \multicolumn{8}{|c|}{ Composition (wt \%) } & \multicolumn{3}{|c|}{ EMTO-CPA calculation } \\
\hline & $\mathrm{Fe}$ & $\mathrm{Cr}$ & $\mathrm{Ni}$ & Mo & V & W & $\mathrm{Nb}$ & $\mathrm{Al}$ & $E(\mathrm{GPa})$ & $H_{\text {mix }}(\mathrm{eV} / \mathrm{at})$ & $G / B$ \\
\hline \multirow{5}{*}{1} & 74 & 20 & 0 & 0 & 0 & 0 & 2 & 4 & 225.4 & 0.0178 & 0.493 \\
\hline & 75 & 20 & 0 & 0 & 0 & 0 & 1 & 4 & 226.5 & 0.0071 & 0.498 \\
\hline & 76 & 19 & 0 & 0 & 0 & 0 & 1 & 4 & 225.6 & 0.0035 & 0.499 \\
\hline & 76 & 20 & 0 & 0 & 0 & 0 & 0 & 4 & 227.7 & -0.0036 & 0.504 \\
\hline & 72 & 22 & 0 & 0 & 0 & 0 & 0 & 6 & 224.9 & -0.0099 & 0.505 \\
\hline \multirow{3}{*}{2} & 74 & 21 & 0 & 0 & 0 & 0 & 0 & 5 & 226.5 & -0.0032 & 0.508 \\
\hline & 71 & 22 & 0 & 0 & 1 & 0 & 0 & 6 & 227.3 & -0.0115 & 0.512 \\
\hline & 69 & 21 & 1 & 0 & 2 & 0 & 0 & 7 & 224.2 & -0.0205 & 0.512 \\
\hline \multirow{5}{*}{3} & 65 & 22 & 4 & 0 & 0 & 0 & 2 & 7 & 219.3 & 0.0086 & 0.496 \\
\hline & 64 & 23 & 4 & 0 & 0 & 2 & 0 & 7 & 222.4 & -0.0004 & 0.506 \\
\hline & 68 & 24 & 1 & 0 & 0 & 0 & 0 & 7 & 226.5 & -0.0114 & 0.514 \\
\hline & 68 & 24 & 1 & 0 & 0 & 0 & 0 & 7 & 226.5 & -0.0114 & 0.514 \\
\hline & 66 & 25 & 0 & 0 & 0 & 0 & 1 & 8 & 227.6 & -0.0073 & 0.517 \\
\hline \multirow{3}{*}{4} & 71 & 20 & 0 & 0 & 2 & 0 & 1 & 6 & 227.7 & -0.0139 & 0.514 \\
\hline & 71 & 22 & 0 & 0 & 0 & 0 & 1 & 6 & 227.8 & -0.0087 & 0.510 \\
\hline & 66 & 25 & 0 & 0 & 0 & 0 & 0 & 9 & 226.0 & -0.0247 & 0.520 \\
\hline
\end{tabular}



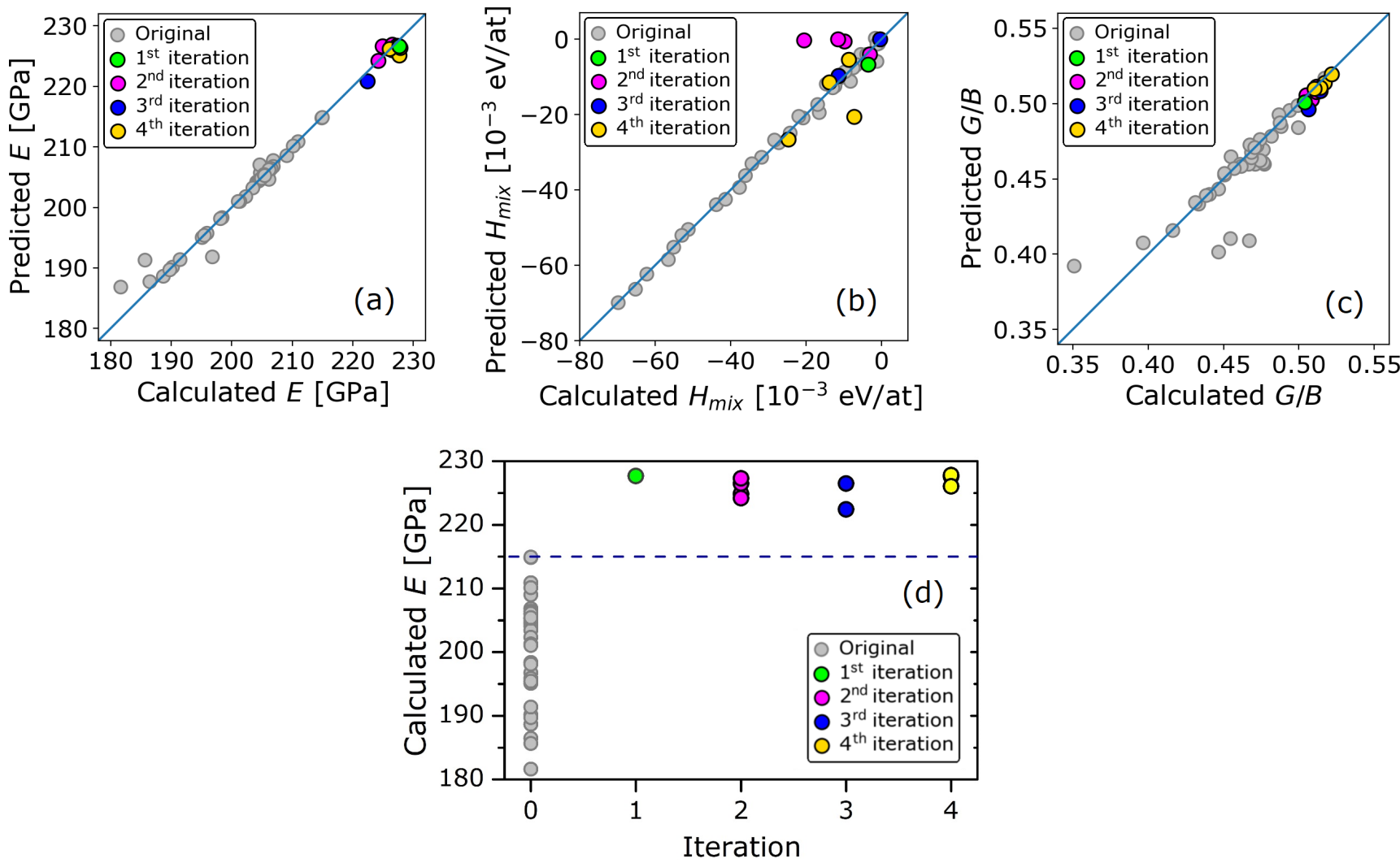

FIG. 4. Predicted target properties $E, H_{\text {mix }}$, and $G / B$ of Fe-Cr-based alloys after four iterations of active learning (a)-(c). Predicted values are plotted versus the calculated values. Scatters plots contain only thermodynamically stable alloys with $H_{\text {mix }}<0$. (d) Calculated Young's moduli $E$ of the alloys from this study suggested via iterative active learning.

when concentrations of $\mathrm{Cr}$ and $\mathrm{Al}$ are high. On the other hand, our literature search confirms that $\mathrm{Fe}-\mathrm{Cr}$ - $\mathrm{Al}$ alloys with concentration 20-25 wt \% $\mathrm{Cr}$ and $5 \mathrm{wt} \% \mathrm{Al}$, the so-called Kanthal alloys, are widely used as commercial materials for high-temperature application [33,34]. The Kanthal alloys are known for their ability to resist oxidation and corrosion at high temperature, which is provided by a slowly growing $a-\mathrm{Al}_{2} \mathrm{O}_{3}$ scale that forms at high temperature [35-39]. High density of $\mathrm{Al}_{2} \mathrm{O}_{3}$ on the surface of alloys in the system $\mathrm{Fe}-\mathrm{Cr}$ $\mathrm{Al}$ prevents them from further oxidation.

\section{CONCLUSIONS}

Considering a task of optimization of stability and elastic properties of multicomponent $\mathrm{Fe}-\mathrm{Cr}$-based alloys, an important material system; e.g., for nuclear energy applications, we have investigated the applicability of the machine learning algorithms for efficient search of unique promising alloys. We have performed an original training of the machine learning models on the dataset of 186 alloys from Ref. [9] considering five empirical features (the atomic radius mismatch $\delta$, average atomic volume $V$, valence electrons concentration $C_{\mathrm{vec}}$, total electronegativity $\chi$, and mixing entropy $S_{\text {mix }}$ ) for the descriptor and targeting the mixing enthalpy $H_{\text {mix }}$, Young's modulus $E$, and the ratio between shear and bulk moduli $G / B$ of the alloys.

The machine learning models obtained in this work have demonstrated the robust prediction capabilities with average cross-validation accuracy over $90 \%$; even though the original dataset contained only 186 samples. The reason for such high accuracy is the extremely homogeneous data in the training set. By extreme homogeneity one can understand that all samples in the training dataset are single-phase bcc alloys, and their properties are calculated within the same EMTO-CPA method and with the same precision. Moreover, the compositions of samples in the original dataset vary with equal steps. This means that one can fit the robust ML models even with a small training set when the quality of data is high.

It should be noted that the training set was limited to only four-component solid solutions, and the use of the machine learning models made it possible to predict the target properties for $\mathrm{Fe}-\mathrm{Cr}$ alloys with up to eight components. This made it possible to consider the contributions from all alloy components and optimize the chemical composition in order to improve the elastic modulus $E$. As a result of the iterative active learning approach, we predicted the three target properties, $E$ modulus, $H_{\text {mix }}$, and $G / B$ for $556578 \mathrm{Fe}-\mathrm{Cr}$-based alloys (see Supplemental Material [32]).

Importantly, we have achieved the main goal of the study and identified the $\mathrm{Fe}-25 \% \mathrm{Cr}-9 \% \mathrm{Al}$ alloy as a highly promising candidate that demonstrates the highest stability with respect to decomposition into the pure alloy components with calculated values of $H_{\text {mix }}=-0.0247 \mathrm{eV} /$ at and the highest calculated value of the Young's modulus, $E=226.0 \mathrm{GPa}$. It turns out that the iterative active learning approach employed in this work has led us to a class of alloys known for their 
high performance in other applications, but not included in the original dataset, demonstrating the high potential of the approach employed in the present study for the design of materials with an optimal combination of targeted properties.

\section{ACKNOWLEDGMENTS}

Financial support from the Knut and Alice Wallenberg Foundation (Wallenberg Scholar Grant No. KAW-2018.0194) and from the Swedish Government Strategic Research Area in Materials Science on Functional Materials at Linköping University, Faculty Grant No. SFO at LiU 200900971 is gratefully acknowledged. First-principles calculations of thermodynamic and mechanical properties of multicomponent alloys were funded by RFBR, Project No. 20-02-00178. The computations were carried out at resources provided by the Swedish National Infrastructure for Computing (SNIC) partially funded by the Swedish Research Council through Grant Agreement No. 2016-07213 and at the supercomputer cluster at NUST "MISIS."
[1] C. Cabet, F. Dalle, E. Gaganidze, J. Henry, and H. Tanigawad, Ferritic-martensitic steels for fission and fusion applications, J. Nucl. Mater. 523, 510 (2019).

[2] G. Ceder and K. Persson, The stuff of dreams, Sci. Am. 309, 36 (2013).

[3] P. Olsson, I. A. Abrikosov, and J. Wallenius, Electronic origin of the anomalous stability of Fe-rich bcc Fe-Cr alloys, Phys. Rev. B 73, 104416 (2006).

[4] T. P. C. Klaver, R. Drautz, and M. W. Finnis, Magnetism and thermodynamics of defect-free Fe-Cr alloys, Phys. Rev. B 74, 094435 (2006).

[5] A. V. Ruban, P. A. Korzhavyi, and B. Johansson, Firstprinciples theory of magnetically driven anomalous ordering in bcc Fe-Cr alloys, Phys. Rev B 77, 094436 (2008).

[6] P. A. Korzhavyi, A. V. Ruban, J. Odqvist, J.-O. Nilsson, and B. Johansson, Electronic structure and effective chemical and magnetic exchange interactions in bcc $\mathrm{Fe}-\mathrm{Cr}$ alloys, Phys. Rev. B 79, 054202 (2009).

[7] A. V. Ponomareva, A. V. Ruban, O. Yu. Vekilova, S. I. Simak, and I. A. Abrikosov, Effect of pressure on phase stability in Fe-Cr alloys, Phys. Rev. B 84, 094422 (2011).

[8] A. V. Ponomareva, A. V. Ruban, B. O. Mukhamedov, and I. A. Abrikosov, Effect of multicomponent alloying with $\mathrm{Ni}, \mathrm{Mn}$ and Mo on phase stability of bcc Fe-Cr alloys, Acta Mater. 150, 117 (2018).

[9] A. V. Ponomareva, M. P. Belov, E. A. Smirnova, K. V. Karavaev, K. Sidnov, B. O. Mukhamedov, and I. A. Abrikosov, Theoretical description of thermodynamic and mechanical properties of multicomponent bcc Fe-Cr-based alloys, Phys. Rev. Materials 4, 094406 (2020).

[10] I. A. Abrikosov, E. E. Son, B. O. Mukhamedov, and A. V. Khvan, Design of materials for nuclear energy applications: Part 1. First-principles calculations and artificial intelligence methods, High Temp. 58, 907 (2020).

[11] W. S. McCulloch and W. Pitts, A logical calculus of the ideas immanent in nervous activity, Bull. Math. Biophys. 5, 115 (1943).

[12] M. Abadi, P. Barham, J. Chen, Z. Chen, A. Davis, J. Dean, M. Devin, S. Ghemawat, G. Irving, and M. Isanrd, TENSORFLOW: A system for large-scale machine learning, in Proceedings of the 12th USENIX Symposium on Operating Systems Design and Implementation "OSDI '16" (USENIX Association, Berkeley, CA, 2016), p. 265

[13] C. Cortes and V. Vapnik, Support-vector networks, Mach. Learn. 20, 273 (1995).
[14] F. Pedregosa, G. Varoquaux, A. Gramfort, V. Michel, B. Thirion, O. Grisel, M. Blondel, P. Prettenhofer, R. Weiss, V. Dubourg et al., SCIKIT-LEARN: Machine learning in PYTHON, J. Mach. Learn. Res. 12, 2825 (2011).

[15] L. Zhang, H. Chen, X. Tao, H. Cai, J. Liu, Y. Ouyang, Q. Peng, and Y. Du, Machine learning reveals the importance of the formation enthalpy and atom-size difference in forming phases of high entropy alloys, Mater. Des. 193, 108835 (2020).

[16] Y. Zhang, Ch. Wen, Ch. Wang, S. Antonov, D. Xue, Y. Bai, and Y. Su, Phase prediction in high entropy alloys with a rational selection of materials descriptors and machine learning models, Acta Mater. 185, 528 (2020).

[17] U. Bhandari, Md. R. Rafi, C. Zhang, and Sh. Yang, Yield strength prediction of high-entropy alloys using machine learning, Mater Today Commun. 26, 101871 (2021).

[18] D. Dai, T. Xu, X. Wei, G. Ding, Y. Xu, J. Zhang, and H. Zhang, Using machine learning and feature engineering to characterize limited material datasets of high-entropy alloys, Comput. Mater. Sci. 175, 109618 (2020).

[19] W. Huang, P. Martin, and H. L. Zhuang, Machine-learning phase prediction of high-entropy alloys, Acta Mater. 169, 225 (2019).

[20] J. Xiong, T. Y. Zhang, and S. Q. Shi, Machine learning prediction of elastic properties and glass-forming ability of bulk metallic glasses, MRS Commun. 9, 576 (2019).

[21] C. H. Hodges and M. J. Stott, Theory of electrochemical effects in alloys, Philos. Mag. 26, 375 (1972).

[22] J. A. Alonso and L. A. Girifalco, Electronegativity scale for metals, Phys. Rev. B 19, 3889 (1979).

[23] A. R. Miedema, P. F. de Chatel, and F. R. de Boer, Cohesion in alloys-fundamentals of a semi-empirical model, Physica B (Amsterdam, Neth.) 100, 1 (1980).

[24] M. Stone, Cross-validatory choice and assessment of statistical predictions, J. R. Stat. Soc. Series B (Methodological) 36, 111 (1974).

[25] L. Vitos, Computational Quantum Mechanics for Materials Engineers: The EMTOMethod and Applications (Springer-Verlag, London, 2007), pp. 83-101.

[26] L. Vitos, I. A. Abrikosov, and B. Johansson, Anisotropic Lattice Distortions in Random Alloys from First-Principles Theory, Phys. Rev. Lett. 87, 156401 (2001).

[27] J. P. Perdew and Y. Wang, Accurate and simple analytic representation of the electron-gas correlation energy, Phys. Rev. B 45, 13244 (1992).

[28] J. P. Perdew, K. Burke, and M. Ernzerhof, Generalized Gradient Approximation Made Simple, Phys. Rev. Lett. 77, 3865 (1996). 
[29] W. Voigt, Ueber die beziehung zwischen den beiden elasticitätsconstanten isotroper körper, Ann. Phys. (Leipzig, Ger.) 274, 573 (1889).

[30] A. Reuss, Berechnung der fließgrenze von mischkristallen auf grund der plastizitätsbedingung für einkristalle, Z. Angew. Math. Mech. 9, 49 (1929).

[31] R. Hill, The elastic behaviour of a crystalline aggregate, Proc. Phys. Soc., Sect. A 65, 349 (1952).

[32] See Supplemental Material at http://link.aps.org/supplemental/ 10.1103/PhysRevMaterials.5.104407 for information on the predicted thermodynamic and elastic properties of multicomponent Fe-Cr-based alloys.

[33] D. Naumenko, V. Kochubey, L. Niewolak, A. Dymiati, J. Mayer, L. Singheiser, and W. J. Quadakkers, Modification of alumina scale formation on FeCrAlY alloys by minor additions of group IVa elements, J. Mater. Sci. 43, 4550 (2008).

[34] W. J. Quadakkers, H. Holzbrecher, K. G. Briefs, and H. Beske, Differences in growth mechanisms of oxide scales formed on ODS and conventional wrought alloys, Oxid. Met. 32, 67 (1989).
[35] F. Liu, H. Götlind, J.-E. Svensson, L.-G. Johansson, and M. Halvarsson, Early stages of the oxidation of a FeCrAlRE alloy (Kanthal AF) at $900^{\circ} \mathrm{C}$ : A detailed microstructural investigation, Corros. Sci. 50, 2272 (2008).

[36] R. Cueff, H. Buscail, E. Caudron, C. Issartel, and F. Riffard, Oxidation behaviour of Kanthal A1 and Kanthal AF at $1173 \mathrm{~K}$ : Effect of yttrium alloying addition, Appl. Surf. Sci. 207, 246 (2003).

[37] C. Badini and F. Laurella, Oxidation of FeCrAl alloy: Influence of temperature and atmosphere on scale growth rate and mechanism, Surf. Coat. Technol. 135, 291 (2001).

[38] G. Berthome, E. N'Dah, Y. Wouters, and A. Galerie, Temperature dependence of metastable alumina formation during thermal oxidation of FeCrAl foils, Mater. Corros. 56, 389 (2005).

[39] H. El Kadiri, R. Molins, Y. Bienvenu, and H. F. Horstemeyer, Abnormal high growth rates of metastable aluminas on $\mathrm{FeCrAl}$ alloys, Oxid. Met. 64, 63 (2005). 